\title{
The carry-over effect does not influence football results
}

\author{
Dries R. Goossens ${ }^{(1,2)}$, Frits C.R. Spieksma ${ }^{(2)}$ \\ (1) PostDoc researcher for Research Foundation - Flanders \\ Corresponding author. E-mail: Dries.Goossens@econ.kuleuven.be \\ (2) Center for Operations Research and Business Statistics (ORSTAT), \\ Faculty of Business and Economics, K.U.Leuven, Belgium
}

\begin{abstract}
In a round robin tournament, it is often believed that each team has an effect on its opponent which carries over to the next game of that opponent. Indeed, if team A plays against team B, and subsequently against team $\mathrm{C}$, A's performance against $\mathrm{C}$ may have been affected by $\mathrm{B}$, and we say that team $\mathrm{C}$ receives a carry-over effect from B. For instance, if team B is a very strong team, then team A could be exhausted and discouraged after this game, which could benefit its next opponent, team C. Clearly, any schedule will lead to carry-over effects. In practice, the perceived influence of carry-over effects has been used as an argument when producing a schedule. In this work, we develop an approach to measure whether carry-over effects have an influence on the outcome of football matches. We apply this method on the highest division in Belgium, using data from over 30 seasons, amounting over 10,000 matches. In our dataset, we find no evidence to support the claim that carry-over effects affect the results, which has major implications for the sporting community with respect to generating fixtures.
\end{abstract}

Keywords: football, carry-over effect, existence, measure, scheduling, fairness 


\section{Introduction}

Most football leagues in Europe are decided by playing a double round robin tournament, i.e. each team plays each other team twice, once at home and once away. Any schedule for a round robin tournament involves an order in which each team meets its opponents. If some team A plays against team B in one round, and against team $\mathrm{C}$ in the next round, we say that team $\mathrm{C}$ receives a carry-over effect (coe) from team B. This carry-over effect is particularly relevant in physical, body-contact sports. For instance, if team B is a very strong, tough-playing side, one can imagine that its opponent, team A, is weakened by injuries or fatigue, which could be an advantage for its next opponent, team C. Or, opposed to this, if team A, confronted with a very strong team B, decides to throw the game, team A will be fit for its next opponent. Moreover, the carryover effect could also be relevant in a strictly psychological interpretation, when team A loses confidence and morale after a severe loss against the strong team B, again to the benefit of their next opponent, team $\mathrm{C}$. The opposite may be true if team B is a weak team. There may also be an influence due to the next opponent of A, say D. For instance, if A meets a strong team D in the next round, they may be more motivated to collect points against their current opponent B. In this case, we can say that team B receives a carry-over effect from team D.

As far as we are aware, no research has been done that involves measuring the significance and/or the size of the carry-over effect for any sport. In other words, this work is the first empirical study on the carry-over effect. The fact that the perceived influence of the carry-over effect on football results was never proven, does not mean its impact on the practice of football should be underestimated. There are several occasions where the carry-over effect was mentioned in popular media, and where the perceived influence of the carry-over effect affected the type of schedules that were implemented. We give two recent examples. 
In the Norwegian highest division (Tippeligean), the 2007 schedule resulted in Brann Bergen playing against Stabaeck's opponent of the previous round in 22 of the 26 rounds. Not only did Stabaeck regularly defeat its opponent, it also had a talented Brazilian player who forced his adversary into making fouls and incurring yellow and/or red cards and resulting suspensions. At the end of the season, the Norwegian media explained the frequent receiving of a carry-over effect from Stabaeck as one of the reasons why Brann Bergen eventually won the league title. This was taken quite seriously by the league organizers, who hired a private company to produce a schedule with more balanced carry-over effects for the next season (Flatberg 2009).

Another occurrence of this phenomenon happened in Belgium, where the 20062007 schedule also had unbalanced carry-over effects. For instance, on 29 of the 34 rounds Beveren played against the team that would confront the top team Anderlecht on the next round. The coach of Beveren claimed that the schedule was unfavorable, since opponents were giving full measure against Beveren, because they realized that in the next game, their chances were slim to collect points. At the end of the season, Beveren relegated to the second division, and has not been able to recover from this setback since. The coach of Beveren was sacked, but mentioned these unbalanced carry-over as an important reason for Beveren's relegation (Geril 2007). This triggered the Belgian national football association to generate a schedule that balances the carry-over effects for the next seasons (Goossens \& Spieksma 2009).

The influence of the carry-over effect is directly related with sports economics in at least two ways. Firstly, the schedule of a football competition is usually the result of a process where many wishes and requests are taken into account; balancing the carry-over effects in a fair way is often one of them. Other requests about fairness are that a team should not start the season playing against all the traditionally strong opponents in a row, or should not end the season 
with a series of away games. Some wishes have a purely practical motivation: two teams share the same stadium, and thus cannot play at home at the same time, or a team cannot play at home when not enough police support is available. Many wishes, though, are about generating revenue: a team wants to play its home games against big teams on a weekend day instead of a weekday, or wants to avoid a succession of home games (see Forrest \& Simmons (2006) who provide a motivation for these wishes). In other words, if the league opts to balance the carry-over effects, this goes at the expense of other wishes. Clearly, in order to produce a high-quality schedule, it is important to understand the background of all wishes, and in particular, to know whether carry-over effects have a serious impact on the final outcome. This knowledge allows decision makers to correctly balance all different types of wishes. Secondly, the revenue of the clubs is determined by the availability of the players, i.e. the number of matches they can play. To decide whether the time between two league matches is long enough to recover from a psychological and physical point of view, it is interesting to measure the influence of the carry-over effect. Indeed, if this influence is substantial, it may provide an argument for more recovery time between consecutive matches, and less matches per year.

In this paper, we want to answer the question whether receiving a carry-over effect influences the result of a game. In other words: does the previous (or next) opponent of your opponent affect your result? An underlying motivation is to be able to tell whether balancing carry-over effects in the schedule is useful. Balancing carry-over effects is the topic of related research; an overview is given in section 2 In section 3 we provide a theoretical framework for this research, including a formal definition of carry-over effects, and an overview of our assumptions. We continue with a section that explains how we measure the influence of carry-over effects. In section 5 , we present and discuss the results, and finally, we formulate some conclusions. 


\section{Related work}

As carry-over effects will unavoidably be present in any schedule, a fair schedule will spread the carry-over effects of each team as evenly as possible over all (other) teams. We define $c_{i j}$ as the number of times team $j$ receives a carry-over effect from team $i$ in the previous round in a schedule; these values can be seen as the elements of a matrix $C$, which we call the carry-over effects matrix. The degree to which the carry-over effects are balanced is typically measured by the so-called carry-over effects value, which is defined as $\sum_{i, j} c_{i j}^{2}$. This measure, together with the problem of finding a schedule for which the carry-over effects value is minimal, was originally proposed by Russell (1980). Table 1 shows an example of a schedule for a single round robin tournament with 6 teams (a), and the corresponding carry-over effects matrix (b). For instance, $c_{41}$, the number of times that team $\mathrm{A}$ receives a carry-over effect from team $\mathrm{D}$, equals 3 , since it happens 3 times that A's opponent played against team D in the previous round. Notice that according to Russell's definition, the carry-over effect from the last round to the first is also counted, although of course in practice this is meaningless. The carry-over effects value for this schedule is 60 , which is actually minimal (Russell 1980).

\begin{tabular}{c|ccccc} 
& 1 & 2 & 3 & 4 & 5 \\
\hline $\mathrm{A}$ & $\mathrm{C}$ & $\mathrm{F}$ & $\mathrm{B}$ & $\mathrm{D}$ & $\mathrm{E}$ \\
$\mathrm{B}$ & $\mathrm{E}$ & $\mathrm{D}$ & $\mathrm{A}$ & $\mathrm{C}$ & $\mathrm{F}$ \\
$\mathrm{C}$ & $\mathrm{A}$ & $\mathrm{E}$ & $\mathrm{F}$ & $\mathrm{B}$ & $\mathrm{D}$ \\
$\mathrm{D}$ & $\mathrm{F}$ & $\mathrm{B}$ & $\mathrm{E}$ & $\mathrm{A}$ & $\mathrm{C}$ \\
$\mathrm{E}$ & $\mathrm{B}$ & $\mathrm{C}$ & $\mathrm{D}$ & $\mathrm{F}$ & $\mathrm{A}$ \\
$\mathrm{F}$ & $\mathrm{D}$ & $\mathrm{A}$ & $\mathrm{C}$ & $\mathrm{E}$ & $\mathrm{B}$
\end{tabular}

(a)

$\begin{array}{llllll}0 & 1 & 3 & 0 & 1 & 0 \\ 0 & 0 & 1 & 3 & 1 & 0 \\ 0 & 0 & 0 & 1 & 1 & 3 \\ 3 & 0 & 0 & 0 & 1 & 1 \\ 1 & 1 & 1 & 1 & 0 & 1 \\ 1 & 3 & 0 & 0 & 1 & 0\end{array}$

(b)

Table 1: Schedule (a) and its carry-over effects matrix (b) for a single round robin tournament with 6 teams

The lowest carry-over effect value we may hope for in a single round robin tournament with $n$ teams is $n(n-1)$. This is the case when all non-diagonal entries of $C$ equal 1 (the diagonal entries always equal zero). A schedule that 
achieves this is called a balanced schedule. Russell (1980) presents an algorithm that results in a balanced schedule when $n$ is a power of two. For other values of $n$, the best known results are by Anderson (1999). Henz, Müller \& Thiel (2004) and Miyashiro \& Matsui (2006) also tackle this problem, but without improving on the results by Anderson. Recently, Guedes \& Ribeiro (2009) presented a weighted variant of the carry-over effects value, taking into account the relative strengths of the teams, and developed a heuristic for the problem to produce a schedule that minimizes this measure. For a more elaborate overview of research on the carry-over effect and sports scheduling in general, we refer to Kendall, Knust, Ribeiro \& Urrutia (2010).

\section{Theoretical framework}

In this section, we develop a formal definition of the carry-over effect. Subsequently, we state a number of assumptions on the influence of team strength and time on the carry-over effect.

Russell (1980) defines the carry-over effect as follows. If team A meets team $\mathrm{B}$ in one match and team $\mathrm{C}$ in the next, then it is reasonable that team A's performance against team $\mathrm{C}$ will have been affected by team $\mathrm{B}$. Team $\mathrm{C}$ is said to receive a carry-over effect due to team B. In Russell's definition, the carry-over effect results from events in the previous round. We generalize this definition in the sense that we also consider the influence of a team's opponent in the next round. This corresponds with the claim by the coach of Beveren that the morale of its opponents was influenced by their next opponent (see section 11. Of course, in this case the influence of the carry-over effect can only be of a psychological kind, whereas in Russell's setting, physical effects can play a role as well. This leads to the following definition.

Definition 1 Given a schedule where in round $r$, team A plays against team $B$, in round $r+1$, team $A$ plays against team $C$, and in round $r+2$, there is a 
match $A$ vs. $D$, we say that team $C$ receives a carry-over effect due to team $B$ in the previous round, and due to team $D$ in the next round. We call team $C$ the receiving team, teams $B$ and $D$ the causing teams, and team $A$ the transferring team.

We do not take into account carry-over effects from the last round to the first or vice versa. Notice that team $\mathrm{C}$ receives two carry-over effects, and that these effects can be positive (if they increase team C's odds against team A) or negative (if they decrease these odds). Our goal is to find out whether carry-over effects influence the outcome of a game. We make no assumption on the size or direction of this influence.

We make the following assumptions concerning the carry-over effect:

- The influence of the carry-over effect depends on the strength of the causing team. This is reasonable since the causing team determines the physical and psychological status of the transferring team. Indeed, a strong causing team may have a completely different effect on e.g. A's morale than a weak causing team.

- We do not take into account the strength of the receiving team, i.e., we assume that carry-over effects from a causing team of given strength is expected to be identical for all receiving teams, regardless of their strength. In other words, we believe that each team is equally susceptible to the influence of the carry-over effect.

- We make no distinction in the strength of the transferring team. The underlying idea is that whereas the strength of the transferring team may determine the influence of the carry-over effect, its impact on the scheduling process is minimal. Indeed, in any round robin tournament, each team will, throughout the season, play against each other team the same number of times. In other words, the strength of the transferring teams is perfectly balanced over the receiving teams in any schedule. 
- If there is a period of 10 days or more between two league games (e.g. because of games between national teams or a winter break), we assume that both the physical and psychological influence of carry-over effects will have faded out. Indeed, the first game of a round is played on a Friday, and the last one on a Sunday. Therefore, to take into account matches from two consecutive rounds, we consider all effects between games that have at most 9 days between them. Apart from that, we make no distinction between the number of days between two matches: a pair of matches with just one day in between is susceptible to an equally strong influence of the carry-over effect as a pair of matches with 9 days in between.

- We assume that the influence of the carry-over effect is constant throughout the season, meaning that for instance, the carry-over effect plays the same role for a match in the beginning of the season as in the end of the season.

In summary, up to 4 carry-over effects can play a role in a match at the same time: carry-over effect from the previous and from the next round for both opponents in the match. We see no way to isolate these effects; there combined occurrence is inherent to any round robin schedule. However, we do think that if we consider all matches with a where similar carry-over effect may play a role, an influence will show if it is present.

\section{Measuring the influence of the carry-over ef- fect}

To measure the influence of the carry-over effect, we use data from the highest division in Belgian football, the so-called Jupiler League, from season 1976-1977 till season 2008-2009, which includes 10,098 games. The Jupiler League consists of 18 teams, playing a double round robin tournament with a mirrored schedule, which is the most common setup in European football leagues. Furthermore, 
Haan, Koning \& van Witteloostuijn (2008) measured the quality difference between the best 4 teams and the other teams in various competitions, and found that for the Jupiler League, this measure is close to average and stable over the last 3 decades. Therefore, it is safe to say that the Jupiler League is a fair representative of football in Europe (see also Goossens \& Spieksma (2010)).

The idea of our approach is to compare the result of each game that is influenced by a carry-over effect with the result that could be expected when no carry-over effects were involved. From the difference between these results, we obtain insight in the significance and the direction of the carry-over effect. In the first subsection, we explain how we arrive at reasonable expected match results, which we can use as a basis of comparison. Finally, we discuss the details of the comparison and set up a statistical significance test.

\subsection{Deriving reasonable expected match results}

In order to determine the influence of the carry-over effect, we need to compare the results of these games with results we could expect under normal circumstances. Therefore, for each game, we need to find a probability distribution that shows what the chances are for a win, a draw, or a loss in an ideal world without carry-over. We will estimate this distribution from the 10,098 games in our dataset.

We reckon that the result of a particular match is determined by the respective strength of the two opponents, and by the home advantage. The strength of a team in a particular season follows from the total number of points the team scored in that season (rather than by their final ranking). Therefore, we assign for each season, each team to one of 10 strength groups, depending on the number of points the team scored in that season. We chose 10 strength groups in order to have sufficiently many observations for each pair of different strength 
groups (using less than 10 groups would not allow us to accurately express the strength of a team). We stick to the "old" rule that a win brings in 2 points, and that 1 point is accorded to a draw: the majority of our data was played under this rule, and its linearity simplifies the analysis below. Ending the season with 20 points or less brings a team in the weakest strength group (i.e. group 1), while scoring at least 53 points will take a team to the strongest strength group for that season (i.e. group 10). The other strength groups are in between, with an interval of 4 points; we use $p_{i}\left(q_{i}\right)$ to denote the lower (upper) bound of the point range of strength group $i$.

This allows us to compute values $w_{i j}\left(d_{i j}, l_{i j}\right)$ as the proportion of games won (drawn, lost) by a home team of strength group $i$ against an away team from strength group $j$. Obviously, not all values are based on an equal number of games, since, for instance, not every season will see a team in the strongest strength group, whereas there are many teams in group 5 . We use $n_{i j}$ to denote the number of games over all seasons on which the proportions between a home team of strength $i$ and an away team of strength $j$ is based. Furthermore, we compute a $10 \times 10$ matrix $A$, with elements $a_{i j}$ being the average result in terms of home team points, i.e. $a_{i j}=2 w_{i j}+d_{i j}$. The average number of points for the away team simply equals $2-a_{i j}$. A graphical representation of matrix $A$ can be found in Figure 1, where the arrows on the horizontal axis go from weak to strong strength groups, and where the vertical axis gives the $a_{i j}$ values. Table 2 show the proportion of home team wins $\left(w_{i j}\right)$.

We see a football match as the outcome of a stochastic process with an underlying probability distribution. The results in the dataset are simply one set of draws from this probability distribution; we will now use the dataset to estimate the probability distributions. Ideally, we would expect a home (away) team to obtain more wins against weaker teams than against stronger teams. Furthermore, the proportion of home (away) losses should be higher against 


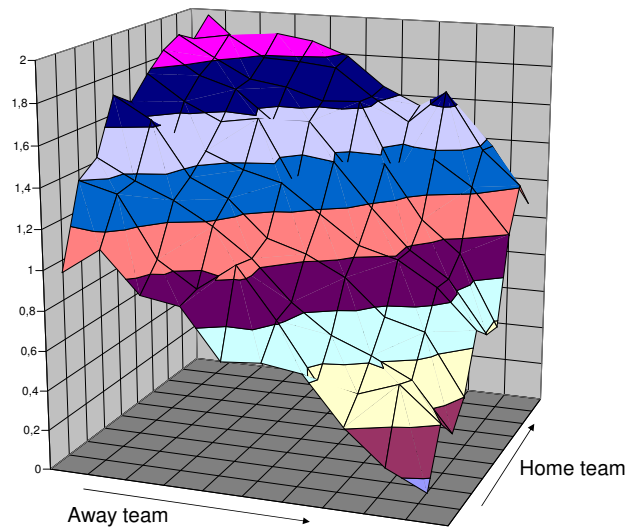

Figure 1: Average home team points without regularity properties (matrix A)

strong teams than against weak teams. We refer to these conditions as regularity properties. It turns out that the vast majority of these regularity properties are satisfied for our dataset, but not all of them. This is witnessed by in Figure 1 where the graph shows several peaks and valleys, and in Table 2, where for instance a home team of strength group 6 has a higher proportion of wins against an away team of strength group 4 than against an away team of the weaker strength group 3. Since these irregularities may well disturb our search for the carry-over effect, we look for more reasonable estimates of the underlying probability distributions. In other words, we want to modify the proportions $w_{i j}$, $d_{i j}$, and $l_{i j}$ as little as possible, but enough to satisfy the regularity properties. For that reason, we developed the following linear optimization model, which uses variables $x_{i j}\left(y_{i j}, z_{i j}\right)$ for the change (positive or negative) we need to make to the dataset proportions $w_{i j}\left(d_{i j}, l_{i j}\right)$ to obtain these properties. The model also uses variables $b_{i j}$ denoting the modified $a_{i j}$ parameters. 


\begin{tabular}{r|rrrrrrrrrr}
$i \backslash j$ & 1 & 2 & 3 & 4 & 5 & 6 & 7 & 8 & 9 & 10 \\
\hline 1 & 0.42 & 0.40 & 0.30 & 0.26 & 0.22 & 0.17 & 0.19 & 0.11 & 0.00 & 0.00 \\
2 & 0.58 & 0.53 & 0.38 & 0.31 & 0.40 & 0.25 & 0.11 & 0.12 & 0.20 & 0.05 \\
3 & 0.60 & 0.54 & 0.50 & 0.45 & 0.38 & 0.29 & 0.19 & 0.17 & 0.12 & 0.05 \\
4 & 0.75 & 0.71 & 0.54 & 0.45 & 0.41 & 0.38 & 0.31 & 0.22 & 0.22 & 0.06 \\
5 & 0.74 & 0.58 & 0.60 & 0.52 & 0.52 & 0.44 & 0.36 & 0.32 & 0.21 & 0.16 \\
6 & 0.82 & 0.73 & 0.68 & 0.70 & 0.47 & 0.56 & 0.44 & 0.34 & 0.28 & 0.11 \\
7 & 0.90 & 0.77 & 0.74 & 0.68 & 0.66 & 0.52 & 0.55 & 0.33 & 0.27 & 0.13 \\
8 & 0.92 & 0.86 & 0.78 & 0.75 & 0.74 & 0.73 & 0.52 & 0.46 & 0.53 & 0.30 \\
9 & 0.90 & 0.94 & 0.85 & 0.84 & 0.79 & 0.70 & 0.68 & 0.70 & 0.56 & 0.43 \\
10 & 0.97 & 0.87 & 0.90 & 0.90 & 0.83 & 0.71 & 0.66 & 0.65 & 0.50 & 0.36
\end{tabular}

Table 2: Proportion of home team wins $\left(w_{i j}\right)$ for each pair of strength groups $i$ and $j$

$\operatorname{minimize} \sum_{i} \sum_{j} n_{i j}\left(\left|x_{i j}\right|+\left|z_{i j}\right|\right)$

subject to

$$
\begin{array}{ll}
w_{i j}+x_{i j} \geqslant w_{i, j+1}+x_{i, j+1} & \forall i \in\{1, . ., 10\}, j \in\{1, . ., 9\} \\
w_{i j}+x_{i j} \leqslant w_{i+1, j}+x_{i+1, j} & \forall i \in\{1, . ., 9\}, j \in\{1, . ., 10\} \\
l_{i j}+z_{i j} \leqslant l_{i, j+1}+z_{i, j+1} & \forall i \in\{1, . ., 10\}, j \in\{1, . ., 9\} \\
l_{i j}+z_{i j} \geqslant l_{i+1, j}+z_{i+1, j} & \forall i \in\{1, . ., 9\}, j \in\{1, . ., 10\} \\
w_{i j}+x_{i j}+d_{i j}+y_{i j}+l_{i j}+z_{i j}=1 & \forall i, j \in\{1, . ., 10\} \\
2\left(w_{i j}+x_{i j}\right)+d_{i j}+y_{i j}=b_{i j} & \forall i, j \in\{1, . ., 10\} \\
p_{i} \leqslant \sum_{j} 34 \frac{n_{i j}\left(b_{i j}\right)+n_{j i}\left(2-b_{j i}\right)}{n_{i j}+n_{j i}} \leqslant q_{i} & \forall i \in\{1, . ., 10\}
\end{array}
$$

The objective function minimizes the deviation from the dataset, weighted by the number of games involved. Notice that changes to the proportion of draws are not included in the objective function. In this way, we sanction a shift of a loss to a win twice as much as changing a game from a draw to a win. Constraints (1) and (2) enforce that a team does not collect more wins against a strong team than against a weak team (for home and away games). Constraints 
(3) and (4) make sure that a team does not lose more often against a weak team than against a strong one (again for home and away games). In this way we have formulated the regularity properties. Constraints (5) make sure that the proportions add up to 1 . Constraints (6) simply define $b_{i j}$ as the number of points a team of strength group $i$ is expected to collect in a home game against a team of strength group $j$. The idea behind the final set of constraints is that the deviation we make from the dataset should not be such that teams end up in another strength group than the one they were originally assigned to. Although this model is non-linear because of the objective function, it can easily be linearized and solved with a standard LP-solver (see e.g. Winston (2004)).

Solving this model gives us a matrix $B$ with elements $b_{i j}$, as depicted in Figure 2 The effect of opponent strength on the match result is documented by the negative slope of the graph. We define $w_{i j}^{\prime}=w_{i j}+x_{i j}, d_{i j}^{\prime}=d_{i j}+y_{i j}$, and $l_{i j}^{\prime}=l_{i j}+z_{i j}$. The proportion of home team wins $\left(w_{i j}^{\prime}\right)$ is given in Table 3 , the proportion of draws $\left(d_{i j}^{\prime}\right)$ can be found in Table 4. The home advantage can be read from the values $w_{i j}^{\prime}$ and $l_{i j}^{\prime}$ for $i=j$, i.e. for matches between teams from identical strength groups. It turns out that $w_{i j}^{\prime}>l_{i j}^{\prime}$ for all $i=j$. Furthermore, the proportions of draws turns out to be more substantial around the diagonal, which is what we may expect when two teams of more or less equal strength meet. However, draws occur more frequently above the diagonal than below, which is again explained by the home advantage. The impact of our model on the proportions was rather limited: $63 \%$ of the proportions were not changed at all; on average proportions changed with only 0.014 percentage points (including a maximal change of $0.167 \mathrm{pp})$. A comparison of tables 2 and 3 illustrates this: most values were not changed, and the changes that were made are very limited.

We claim that the proportions $w_{i j}^{\prime}, d_{i j}^{\prime}$, and $l_{i j}^{\prime}$ provide a reasonable probability distribution for the outcome of a game of a strength group $i$ home team versus 


\begin{tabular}{r|rrrrrrrrrr}
$i \backslash j$ & 1 & 2 & 3 & 4 & 5 & 6 & 7 & 8 & 9 & 10 \\
\hline 1 & 0.42 & 0.40 & 0.30 & 0.26 & 0.22 & 0.17 & 0.12 & 0.11 & 0.00 & 0.00 \\
2 & 0.58 & 0.53 & 0.38 & 0.31 & 0.31 & 0.25 & 0.12 & 0.12 & 0.12 & 0.05 \\
3 & 0.60 & 0.54 & 0.50 & 0.45 & 0.38 & 0.29 & 0.19 & 0.17 & 0.12 & 0.05 \\
4 & 0.75 & 0.71 & 0.54 & 0.45 & 0.41 & 0.38 & 0.31 & 0.22 & 0.22 & 0.06 \\
5 & 0.75 & 0.71 & 0.60 & 0.52 & 0.52 & 0.44 & 0.36 & 0.32 & 0.22 & 0.13 \\
6 & 0.82 & 0.73 & 0.68 & 0.68 & 0.52 & 0.52 & 0.44 & 0.34 & 0.28 & 0.13 \\
7 & 0.90 & 0.77 & 0.74 & 0.68 & 0.66 & 0.52 & 0.52 & 0.34 & 0.28 & 0.13 \\
8 & 0.92 & 0.86 & 0.78 & 0.75 & 0.74 & 0.70 & 0.52 & 0.52 & 0.52 & 0.30 \\
9 & 0.92 & 0.92 & 0.85 & 0.84 & 0.79 & 0.70 & 0.66 & 0.66 & 0.56 & 0.36 \\
10 & 0.97 & 0.92 & 0.90 & 0.90 & 0.83 & 0.71 & 0.66 & 0.66 & 0.56 & 0.36
\end{tabular}

Table 3: Proportion of home team wins $\left(w_{i j}^{\prime}\right)$ after applying the model, for each pair of strength groups $i$ and $j$

\begin{tabular}{r|rrrrrrrrrr}
$i \backslash j$ & 1 & 2 & 3 & 4 & 5 & 6 & 7 & 8 & 9 & 10 \\
\hline 1 & 0.30 & 0.33 & 0.34 & 0.38 & 0.22 & 0.26 & 0.30 & 0.19 & 0.24 & 0.14 \\
2 & 0.25 & 0.30 & 0.29 & 0.32 & 0.32 & 0.30 & 0.30 & 0.26 & 0.25 & 0.18 \\
3 & 0.28 & 0.29 & 0.29 & 0.29 & 0.28 & 0.26 & 0.32 & 0.30 & 0.28 & 0.17 \\
4 & 0.21 & 0.21 & 0.28 & 0.37 & 0.28 & 0.27 & 0.31 & 0.32 & 0.26 & 0.29 \\
5 & 0.21 & 0.21 & 0.26 & 0.33 & 0.29 & 0.30 & 0.33 & 0.36 & 0.44 & 0.35 \\
6 & 0.15 & 0.20 & 0.19 & 0.19 & 0.29 & 0.29 & 0.30 & 0.33 & 0.38 & 0.35 \\
7 & 0.07 & 0.19 & 0.20 & 0.19 & 0.22 & 0.29 & 0.29 & 0.42 & 0.38 & 0.35 \\
8 & 0.05 & 0.10 & 0.16 & 0.19 & 0.14 & 0.18 & 0.29 & 0.29 & 0.18 & 0.40 \\
9 & 0.05 & 0.04 & 0.10 & 0.11 & 0.15 & 0.23 & 0.16 & 0.16 & 0.25 & 0.43 \\
10 & 0.03 & 0.07 & 0.09 & 0.06 & 0.13 & 0.23 & 0.23 & 0.23 & 0.30 & 0.43
\end{tabular}

Table 4: Proportion of draws $\left(d_{i j}^{\prime}\right)$ after applying the model, for each pair of strength groups $i$ and $j$

an away team of strength group $j$, which can be used a basis of comparison ${ }^{1}$. It is true that these proportions are based on a dataset which is obviously not free of carry-over effects. However, we think this is inevitable, since only very few games are not preceded or followed within 10 days by another game featuring one of the contenders. Furthermore, the size of the dataset is such that these positive and negative effects cancel out when data is aggregated in strength groups.

\footnotetext{
${ }^{1}$ Notice that the analysis in section 4.2 can also be done with the original proportions $w_{i j}$, $d_{i j}$, and $l_{i j}$ as a basis of comparison, which leads to very similar results for the influence of the carry-over effect.
} 


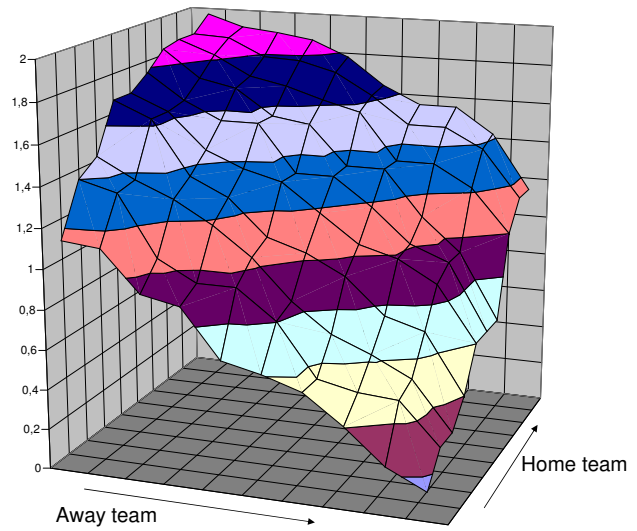

Figure 2: Average home team points with regularity properties (matrix B)

\subsection{Comparison and significance test}

We distinguish between carry-over effects from the previous and the next round, and we estimate the influence of carry-over effects for causing teams from each of the ten strength groups. For each of these carry-over effects, we compare the actual result with the expected result as given by the matrix $B$. Notice that the matches where a team receives a carry-over effect from a team belonging to some strength group $s$ can be between teams from any strength group. For instance, Table 5 shows the relevant data for 5 games from season 1976-1977 where a team receives a carry-over effect from a causing team from strength group 1 (i.e. a weak team).

The first column gives the team that receives the carry-over effect, with between brackets its strength group in that season. The second column gives the transferring team, i.e. the opponent opponent of the receiving team, who played a team from strength group 1 in its previous match. The receiving team can be the home side or the away side (column 3). For the first row, the re- 


\begin{tabular}{rrr|rr|rrr} 
team & opponent & $\mathrm{h} / \mathrm{a}$ & result & $\bar{b}_{i j}$ & $\bar{w}_{i j}^{\prime}$ & $\bar{d}_{i j}^{\prime}$ & $\bar{l}_{i j}^{\prime}$ \\
\hline KOR[4] & WIN[4] & away & 2 & 0.734 & 0.181 & 0.372 & 0.447 \\
WIN[4] & ANT[5] & home & 0 & 1.094 & 0.408 & 0.278 & 0.314 \\
AND[8] & CER[5] & home & 2 & 1.617 & 0.740 & 0.137 & 0.123 \\
WAR[5] & BER[3] & home & 2 & 1.449 & 0.597 & 0.255 & 0.148 \\
BEV[4] & RWD[7] & away & 1 & 0.445 & 0.128 & 0.189 & 0.683 \\
\hline & & sum: & 7 & 5.33 & 2.054 & 1.231 & 1.715
\end{tabular}

Table 5: List of games with a team receiving carry-over from a strength group 1 team

ceiving team is Kortrijk (KOR), playing an away game against the transferring team Winterslag (WIN), both belonging to strength group 4. The following column gives the actual result of the game, in terms of points collected by the receiving team. The next column gives the points that Kortrijk would normally obtain in an away game against Winterslag given that they are both in the 4th strength group $\left(\bar{b}_{i j}=b_{i j}\right.$ if our team plays at home and $\bar{b}_{i j}=2-b_{i j}$ if our team plays away; similarly for $\bar{w}_{i j}^{\prime}, \bar{d}_{i j}^{\prime}$, and $\bar{l}_{i j}^{\prime}$ ). The last three columns give the probabilities for the outcomes that the receiving team wins (column 6), draws (column 7), or loses (column 8) this game, as derived in the previous subsection. For this example, we easily compute that the receiving team scores on average 0.334 points more per game than expected. To test whether the actual results differ significantly from the probability distribution we expect, we perform a chi-square test. For this case (obviously with insufficient observations), the test (see Table 6) does not allow us to conclude that the carry-over effect has a significant influence.

\begin{tabular}{lrrr} 
& win & draw & loss \\
\hline \multicolumn{1}{c}{ actual number of observations } & 3.000 & 1.000 & 1.000 \\
estimated number of observations & 2.054 & 1.231 & 1.715 \\
\hline$\chi^{2}=0.78$ & & & \\
$\chi_{0.99}^{2}=9.21($ d.f. $=2)$ & & & \\
$p$-value $=0.68$ & & &
\end{tabular}

Table 6: Chi-square test for the example in Table 5

Notice that our approach assumes that the total number of points obtained by a team at the end of the season is not substantially influenced by the carry-over 
effect. If this is not the case, then carry-over effects may be underestimated. In many countries (e.g. Spain, Turkey, Russia), the canonical one-factorization (see De Werra (1980)) is used to schedule the league. Until the season 2007-2008, this was also the case in Belgium (Goossens \& Spieksma 2009). A particular feature of this schedule is that it has a large carry-over effects value. Consequently, for some teams the season's schedule is completely dominated by the carry-over effect received from one particular team. For instance, the Beveren coach could claim that the reason that Beveren collected only 20 points and ended up in the lowest strength group is precisely the fact that they receive carry-over effects from Anderlecht 29 times in 34 rounds. Therefore, their results should not be compared with results from the lowest strength group, but with a higher one, which would show an increased influence of carry-over effects. A similar reasoning goes for teams that may have been benefited by the carry-over effect. It is difficult to predict in which strength group a team would have ended up without such pronounced carry-over effect. Therefore, our analysis will only take into account teams that receive carry-over effects at most 6 times from the same team. Indeed, in this case we can safely assume that positive and negative carry-over effects will cancel out each other over a whole season, and that the carry-over effect will not influence the strength group a team ends up in. We chose 6 here as a compromise between on the one hand having enough games to do a statistical analysis, and on the other hand reducing the effect described above.

\section{Results}

Table 7 summarizes the results obtained with our approach for the carry-over effect in the Belgian Jupiler League. The values in the second column are the average number of points gained per game because of receiving carry-over effect from the previous round, by a causing team from the strength group $s$ corre- 
sponding to the row. The table shows e.g. that a team may expect to collect 0.058 points less in a match against an opponent that played against a team from strength group 3 in its previous match, than in normal circumstances. The third column gives the $p$-values for the previously discussed chi-square test. The number of carry-over effects these values are based on are mentioned in the fourth column. Similar values are presented for carry-over effects from the next round. As explained in the previous section, we only took into account teams that receive carry-over effects at most 6 times from the same team. Although this allows us to assume that the carry-over effect did not influence a team's strength group, it drastically reduces the set of matches we can use. For reasons of completeness, we repeated our analysis on the complete dataset; the results are given in Table 8.

\begin{tabular}{r|rrr|rrr} 
& \multicolumn{4}{|c}{ previous round } & \multicolumn{4}{c}{ next round } \\
$\mathrm{s}$ & extra points & p-value & \multicolumn{1}{r}{$\sharp$ effects } & extra points & p-value & $\sharp$ effects \\
\hline 1 & 0.020 & 0.875 & 56 & 0.084 & 0.737 & 64 \\
2 & -0.056 & 0.528 & 64 & 0.008 & 0.939 & 69 \\
3 & -0.058 & 0.390 & 161 & 0.077 & 0.278 & 166 \\
4 & 0.007 & 0.837 & 167 & -0.042 & 0.799 & 169 \\
5 & 0.036 & 0.735 & 134 & -0.065 & 0.491 & 145 \\
6 & 0.096 & 0.303 & 124 & -0.029 & 0.057 & 105 \\
7 & -0.071 & 0.108 & 96 & -0.040 & 0.586 & 113 \\
8 & -0.144 & 0.029 & 44 & 0.008 & 0.877 & 61 \\
9 & 0.080 & 0.510 & 50 & -0.016 & 0.937 & 55 \\
10 & 0.030 & 0.746 & 51 & -0.083 & 0.696 & 42
\end{tabular}

Table 7: Influence of carry-over effects from the previous and the next round by causing teams from strength group $s$, filtered for teams with schedules balanced w.r.t. carry-over effects

Tables 7 and 8 show that the $p$-values are not small enough to conclude that the distribution of wins, draws and losses is any different from the distribution we would expect if no carry-over effects were present. Moreover, the influence of the carry-over effects does not seem to depend on the strength of the causing team. Overall, the tables allow us to conclude that carry-over effects do not substantially influence match results in Belgium's highest football league. We 


\begin{tabular}{r|rrr|rrr} 
& \multicolumn{3}{|c}{ previous round } & \multicolumn{4}{c}{ next round } \\
$\mathrm{s}$ & extra points & p-value & \multicolumn{1}{r}{ \#effects } & extra points & p-value & $\sharp$ effects \\
\hline 1 & -0.006 & 0.550 & 1016 & 0.020 & 0.822 & 1002 \\
2 & -0.010 & 0.231 & 1202 & 0.015 & 0.850 & 1198 \\
3 & -0.010 & 0.956 & 2986 & -0.010 & 0.696 & 2986 \\
4 & -0.010 & 0.300 & 2933 & 0.000 & 0.897 & 2883 \\
5 & 0.000 & 0.907 & 2139 & -0.010 & 0.785 & 2130 \\
6 & 0.022 & 0.575 & 1717 & -0.013 & 0.796 & 1725 \\
7 & 0.021 & 0.113 & 1602 & 0.040 & 0.229 & 1620 \\
8 & -0.017 & 0.024 & 900 & 0.025 & 0.392 & 895 \\
9 & -0.001 & 0.927 & 917 & 0.010 & 0.923 & 910 \\
10 & 0.004 & 0.637 & 730 & -0.013 & 0.487 & 739
\end{tabular}

Table 8: Influence of carry-over effects from the previous and the next round by causing teams from strength group $s$, based on the complete dataset

did find one significant $p$-value in both tables, indicating that a team that plays against an opponent that played against a team of strength group 8 in its previous game collects on average 0.144 points less than expected. When we break down this figure depending on the result of the transferring team against the causing team of strength group 8 , we obtain the influence of the carry-over effect in Table 9 (the number between brackets is the number of carry-over effects it is based on).

\begin{tabular}{rrr} 
win & draw & loss \\
\hline$-0.060(9)$ & $0.160(10)$ & $-0.296(25)$
\end{tabular}

Table 9: Influence of carry-over effects received from teams from strength group 8 , depending on the result of the transferring team against this causing team

Table 9 shows that the matches lost against the team of strength group 8 are responsible for the influence of the carry-over effect. A possible explanation may be that an unexpected loss causes teams to start the next match fully focused, and with determination to show what they are worth. Indeed, a confrontation against a team of strength 8 usually results in a loss, however, because these teams are not perceived as absolute top teams, this loss may generally not be expected. For instance, the yearly phenomenon of an average team that surprises itself and its opponents with an exceptional season typically results in a strength group 8 team. This effect does not show for weaker teams $(s<8)$, 
because there, the chances of losing are smaller. For stronger teams $(s>8)$, i.e. the first or second in the league, losing is simply perceived as normal, and results in no extra motivation.

Until now, we only looked at how the carry-over effect may affect the outcome of a game. However, it may well be the case that this influence is too small to be noticed when looking at the results. Indeed, the team that would win in a world without carry-over effects may still win when carry-over effects play a role, but only just. If the carry-over effect results in a more narrow win, or a more distinct loss, we should be able to tell through the goal difference. Therefore, we repeat our analysis, but this time based on goal difference instead of points. The results are given in Table 10. We make a distinction between carry-over effects from the previous and the next round, and again only use the matches of those teams that receive carry-over effects at most 6 times from the same team. The entries should be interpreted as the difference in goal difference due to the carry-over effect (second column), followed by the $p$-values for the chi-square test (third column). The number of carry-over effects on which this is based can be found in the corresponding column in Table 7 .

The study on goal difference confirms the results in Table 7. Again, the carryover effect received from the previous round by causing teams of strength group 8 is the only one with a small but significant influence. All other $p$-values don't indicate that the distribution of goal differences is different when carry-over effects are involved. Moreover, Table 10 produces no evidence that the strength of the causing team determines the size or direction of the effect. 


\begin{tabular}{r|rrrr} 
& \multicolumn{2}{|c}{ previous round } & \multicolumn{2}{c}{ next round } \\
$\mathrm{s}$ & extra goals & p-value & extra goals & p-value \\
\hline 1 & -0.246 & 0.748 & 0.206 & 0.446 \\
2 & -0.131 & 0.521 & 0.035 & 0.922 \\
3 & -0.157 & 0.769 & 0.145 & 0.833 \\
4 & -0.005 & 0.778 & -0.297 & 0.561 \\
5 & 0.028 & 0.543 & -0.117 & 0.835 \\
6 & 0.180 & 0.912 & 0.060 & 0.078 \\
7 & -0.056 & 0.488 & -0.112 & 0.832 \\
8 & -0.173 & 0.043 & -0.100 & 0.685 \\
9 & -0.154 & 0.393 & -0.144 & 0.800 \\
10 & 0.175 & 0.141 & 0.051 & 0.708
\end{tabular}

Table 10: Influence of carry-over effects from the previous and the next round by causing teams from strength group $s$ on goal difference

\section{Conclusion}

We designed an approach to measure the influence of carry-over effects. We looked at carry-over effects from the previous and the next round within Belgium's highest football league. To obtain a basis of comparison, we estimated a distribution that gives the chance of a win, a draw, or a loss, depending on the strength of the team and the home advantage.

We found that the influence of carry-over on the result and the goal difference of a match is negligible. The distribution of results and goal differences of carryover influenced matches does not significantly differ from the distribution we obtained as a basis of comparison.

Given the assumptions that we made, we conclude that in general, a football team cannot rightfully claim to be at a disadvantage because of a schedule that does not balance carry-over effects. This does however not exclude that there may exist specific circumstances where the carry-over effect may have an influence. For instance, a team with a small number of players may be more susceptible to the carry-over effect than a team with a lot of players, which can more easily deal with injuries or suspensions, or even simply put a completely fresh team on the pitch after a difficult match. Furthermore, it remains open for 
future research whether a carry-over effect resulting from matches played in European competitions (Champions League, Europa League) influences matches in the domestic league. However, this paper shows that - at least in the setting we studied - carry-over effects are not an explanation for winning or losing a match, let alone for winning the title or for relegating. Thus, balancing carryover effects does not seem to improve fairness, and apparently, the time between two matches is sufficiently long to recover both from a physical and psychological point of view.

\section{Acknowledgement}

The authors wish to thank prof. dr. Willy Gochet for his valuable comments on our approach to measure carry-over effects, and the referees for their remarks.

\section{References}

Anderson, I. (1999). Balancing carry-over effects in tournaments, in F. Holroyd, K. Quinn, C. Rowley \& B. Webb (eds), Combinatorial designs and their applications, Vol. 403 of Research Notes in Mathematics, Chapman \& Hall/CRC, pp. 1-16.

De Werra, D. (1980). Geography, games and graphs, Discrete Applied Mathematics 2(4): 327-337.

Flatberg, T. (2009). Scheduling the topmost football leagues of Norway, in EURO XXIII: Book of abstract of the 23rd European Conference on Operational Research, Bonn, Germany, p. 240.

Forrest, D. \& Simmons, R. (2006). New issues in attendance demand: The case of the English football league, Journal of Sports Economics 7(3): 247-266. 
Geril, J. (2007). Ons budget voor transfers? Nul komma nul euro, Het Nieuwsblad, February 2nd (VUM) [Dutch].

Goossens, D. \& Spieksma, F. (2009). Scheduling the Belgian soccer league, Interfaces 39(2): 109-118.

Goossens, D. \& Spieksma, F. (2010). Soccer schedules in Europe: an overview, FBE Research Report KBI 1011, K.U.Leuven.

Guedes, A. \& Ribeiro, C. (2009). A hybrid heuristic for minimizing weighted carry-over effects in round robin tournaments, in MISTA'09: Proceedings of the 4th Multidisciplinary International Conference on Scheduling: Theory and Applications, Dublin, Ireland.

Haan, M., Koning, R. \& van Witteloostuijn, A. (2008). Competitive balance in national European soccer competitions, in J. Albert \& R. Koning (eds), Statistical thinking in sports, Chapman \& Hall/CRC, pp. 63-75.

Henz, M., Müller, T. \& Thiel, S. (2004). Global constraints for round robin tournament scheduling, European Journal of Operational Research 153: 92 101.

Kendall, G., Knust, S., Ribeiro, C. \& Urrutia, S. (2010). Scheduling in sports: An annotated bibliography, Computers and Operations Research 37: 1-19.

Miyashiro, R. \& Matsui, T. (2006). Minimizing the carry-over effects value in a round-robin tournament, in PATAT' 06: Proceedings of the 6th International Conference on the Practice and Theory of Automated Timetabling, Brno, Czech Republic, pp. 402-405.

Russell, K. (1980). Balancing carry-over effects in round robin tournaments, Biometrika 67(1): 127-131.

Winston, W. L. (2004). Operations research: applications and algorithms, Brooks/Cole, Thomson Learning, Inc. 